\title{
Design of tensegrity structures using parametric analysis and stochastic search
}

\author{
Landolf Rhode-Barbarigos ${ }^{1, *}$, Himanshu Jain ${ }^{2}$, Prakash Kripakaran ${ }^{1}$, Ian F.C. Smith ${ }^{1}$
}

\author{
${ }^{1}$ Applied Computing and Mechanics Laboratory, ENAC-IS-IMAC, Station 18, Ecole \\ Polytechnique Fédérale de Lausanne (EPFL), Lausanne CH-1015, Switzerland. \\ ${ }^{2}$ Department of Civil Engineering, IIT Bombay, India. \\ * Corresponding author. Tel: +41 2169363 72, Fax: +41 2169347 48, Email: \\ landolf-giosef.rhode-barbarigos@epfl.ch
}

\begin{abstract}
Tensegrity structures are lightweight structures composed of cables in tension and struts in compression. Since tensegrity systems exhibit geometrically nonlinear behavior, finding optimal structural designs is difficult. This paper focuses on the use of stochastic search for the design of tensegrity systems. A pedestrian bridge made of square hollow-rope tensegrity ring modules is studied. Two design methods are compared in this paper. Both methods aim to find the minimal cost solution. The first method approximates current practice in design offices. More specifically, parametric analysis that is similar to a gradient-based optimization is used to identify good designs. Parametric studies are executed for each system parameter in order to identify its influence on response. The second method uses a stochastic search strategy called Probabilistic Global Search Lausanne (PGSL). Both methods provide feasible configurations that meet civil engineering criteria of safety and serviceability. Parametric studies also help in defining search parameters such as appropriate penalty costs to enforce constraints while optimizing using stochastic search. Traditional design methods are useful to gain an understanding of structural behavior. However, due to the many local minima in the solution space, stochastic search strategies find better solutions than parametric studies.
\end{abstract}

\section{Keywords}

Tensegrity; Bridge; Structural design; Optimization; Stochastic search.

\section{Introduction}

Tensegrity structures are spatial structural systems that contain tensile and compression elements. They are composed of struts and cables with reticulated connections. Thus, tensegrity structures are a subclass of cable structures with the important property that tensile forces are not anchored [1]. Their stability is based on a self-equilibrated self-stress state. Although, tensegrity systems have been studied since 1961 [2], the most complete definition was given by Motro in 2003 [3]: "A tensegrity system is a system in stable self-equilibrated state comprising a discontinuous set of compressed components inside a continuum of tensioned components". These two sets of components combine to form a stable system when an initial self-stress is imposed. Researchers have now broadened the domain of tensegrity systems to include those 
that have continuous compression members. Continuous compression members increase the bending stiffness of the system [4].

Tensegrity structures are relatively lightweight systems compared with other structural systems that offer the same load bearing capacity. Their ability to respond by distributing the effect of loading into many members makes tensegrity structures economical in terms of material cost. Therefore, tensegrity systems have potential to be good structural systems for bridges. Additionally, tensegrity structures can integrate multiple functions for the same elements. For example, cables are loaded elements that can also be used in an active control system [5-7].

While tensegrity systems offer many structural and functional benefits, they are seldom used in practice due to complexities in analysis, design and fabrication. The analysis of tensegrity systems should take into account the following factors, which are either neglected or absent in common civil engineering structures such as frames and trusses.

- A form-finding step is necessary to identify the initial topology of a tensegrity structure on the basis of an initial self-equilibrated self-stress. This step may involve analytical or experimental methods. Form-finding methods have been extensively studied; a review of methods can be found in [5]. These methods may also be used for analysis. Masic et al. [6] proposed a systematic procedure for form finding that includes design considerations such as element strength.

- Tensegrity action involves large displacements and therefore, an accurate analysis of these systems usually requires consideration of geometrical nonlinearity.

- Tensegrity systems have closely coupled behavior [7]: the behavior of the structure cannot be predicted from analyzing the behavior of individual components.

- Tensegrity structures may be kinematically indeterminate.

Since the analysis of tensegrity systems is not straightforward and only a few tensegrity structures have been constructed, no generally agreed guidelines for design exist. Previous studies on simple tensegrity structures have revealed the importance of many parameters such as the level of self-stress or the rigidity ratio between struts and cables [8]. For example, self-stress levels may be adjusted to increase load-bearing capacity [2]. Quirant et al [9] proposed a design procedure for tensegrity grids and applied it for the construction of a double layer tensegrity grid covering a surface of $81 \mathrm{~m}^{2}$. This procedure has, however, not been generalized for other applications.

In practice, an iterative approach similar to a gradient-based search is employed for design. The method begins with a trial solution and then design parameters are modified depending upon constraint violations to arrive at a feasible solution. For example, engineers designing tensegrity systems may gradually increment the areas of struts and cables to meet the force requirements. However, additional criteria such as the elimination of slack cables can make it significantly difficult to find a feasible solution. The adjustments to design parameters are based upon the influence of parameters that are contained in objectives and constraints. The assumption is that the search space has a single minimum cost solution and individually adjusting the design parameters leads to this minimum. However, this assumption is seldom valid as design spaces have multiple local minima. Gradient-based search often results in solutions that are only locally optimal. Also, it is often of interest to generate a number of good designs so that designers can select preferred solutions using design criteria that are not modeled explicitly in the objective function. 
Researchers [10] have studied extensively the use of stochastic optimization methods such as genetic algorithms and simulated annealing to find the global minimum for the design of structural systems such as frames [11, 12] and trusses [13, 14]. However, very little research has examined the use of these optimization methods for the design of tensegrity systems. Tensegrity structures involve a higher level of complexity due to their nonlinear behavior and can benefit from the use of design optimization [15]. Finding optimal member sizes for struts and cables such that the structure satisfies all design criteria is difficult. Stochastic search explores the search space by generating and testing many solutions to find good solutions [16]. Paul et al [17] proposed an algorithm using genetic algorithms for the determination of connectivity patterns which lead to stable tensegrity systems. However, their algorithm is primarily for form-finding and can be computationally expensive due to the number of solutions that need to be evaluated.

In this paper, a pedestrian bridge composed of tensegrity ring modules is designed using two methods. The first method simulates traditional design through parametric analyses, while the second uses a direct stochastic search called PGSL (Probabilistic Global Search Lausanne). PGSL is a stochastic sampling method for global optimization that has been shown to give better performance than other stochastic optimization methods for engineering tasks such as configuration, diagnosis and control [16, 18-20].

\section{Tensegrity ring modules}

Tensegrity ring modules are elementary tensegrity systems that first appeared in Anthony Pugh's book “An introduction to tensegrity" [2]. Their design involves a circuit of compressed components that enhances bending stiffness [4]. Ring modules have also been shown to be deployable. Due to these features, assemblies of tensegrity ring modules are potentially viable structural systems for bridges.

Tensegrity ring modules are easy to conceive [21]. The modules studied in this paper are created using the procedure below.

a. For a n-sided polygonal cell the first step is the construction of a straight prism with n-sided polygonal bases. This prism is used only for developing the topology of the module and is not a part of the module.

b. Diagonal struts are added to all rectangular faces of the cell. Rotational symmetry about the vertical axis passing through the centers of top and bottom faces of the prism is required as shown in Figure 1a.

c. Additional nodes referred to as $\mathrm{x}$-nodes are now added. Consider a circumscribing circle that is mid-way between the top and bottom faces of the prism. In this study, x-nodes are chosen on the middle of the arc corresponding to each face of the cell. Each of these nodes is then connected to two base nodes, one each at the top and bottom faces of the prism, by adding two new struts, called non-diagonal struts (Figure 1b). As in the previous step, base nodes are chosen such that the resulting strut arrangement ensures rotational symmetry.

d. Tensegrity rings contain cables that can be separated into two sets: the layer cables and the $\mathrm{x}$-cables. The layer cables connect the nodes at the top and bottom prism faces (Figure 1c).

e. The $\mathrm{x}$-cables are positioned around the module connecting the $\mathrm{x}$-nodes with the four nodes of each rectangular face of the prism (Figure 1d). 


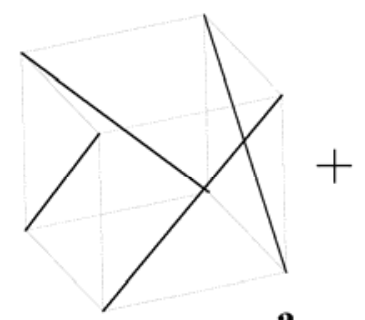

a

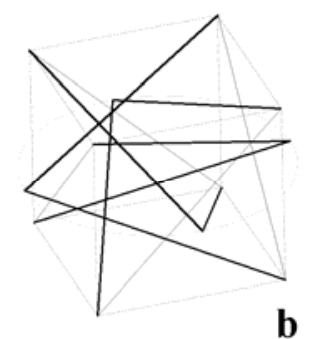

b
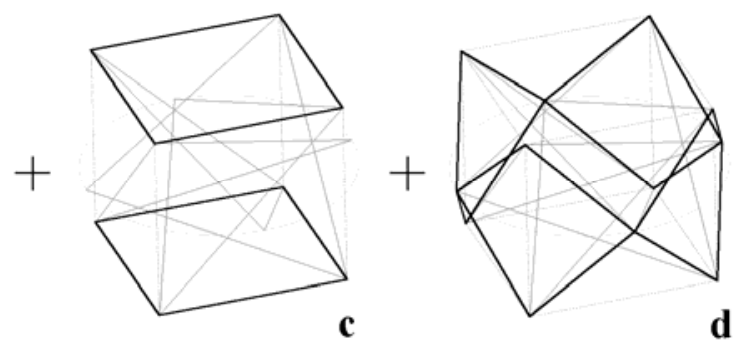

Figure 1: Conception of a square tensegrity ring module: a) diagonal struts, b) non-diagonal struts, c) layer cables and d) x-cables

\section{Hollow rope pedestrian bridge}

Tensegrity ring modules can be assembled together to form a structural system resembling a hollow rope. In this study, the system is used for a pedestrian bridge. Four identical modules are connected base to base to span a bridge. A peculiarity of these modules is that multiple compression elements (struts) meet at a single node.

For a given geometry, these modules can be characterized by three parameters [21]: their length $L$; their outer diameter $D$ and their thickness $t$ (Figure 2). These parameters are sufficient to define a hollow tube containing all elements of the tensegrity ring. The length $L$ is the total stretched length of the module. The outer diameter $D$ is the diameter of the circle circumscribing the base of the module. The thickness $t$ corresponds to the difference between the outer diameter $D$ and the inner diameter $d$. The inner diameter $d$ is the diameter of the central hollow space. This space is used for traversing the bridge.
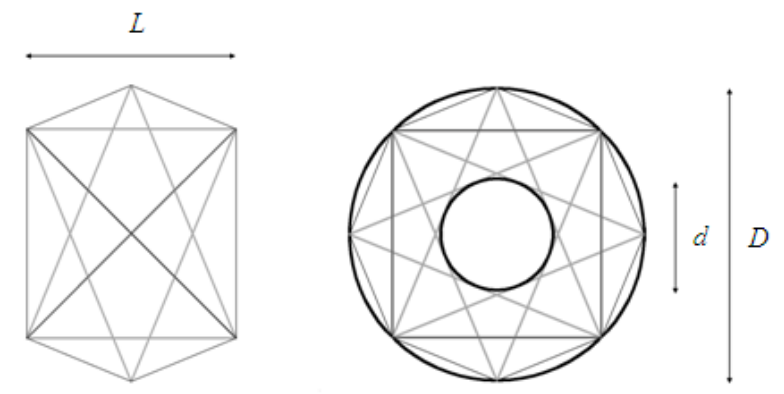

Figure 2: Parameters characterizing ring modules: side view (left) and front view (right)

Several polygonal geometries are possible for the ring module. A square geometry is chosen in this study (Figure 3). In this topology, each diagonal strut is the third side of a triangular circuit formed by two non-diagonal struts starting from a node on the face opposite to the diagonal. In total a square module contains four triangular circuits. This geometry is similar to the one originally proposed by Pugh [2, 21]. 


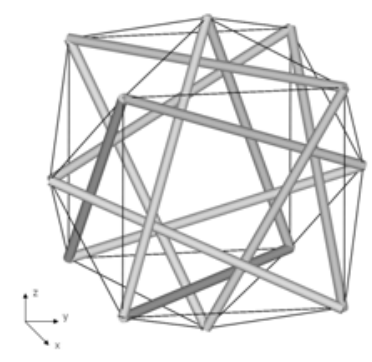

Figure 3: The square ring module

Tensegrity systems are characterized by a number of infinitesimal mechanisms and a number of independent states of self-stress for a specific pre-stressed configuration. Self-stress states stiffen the structure and stabilize infinitesimal mechanisms. Both the number of infinitesimal mechanisms and the number of independent states of self-stress can be determined through a Singular Value Decomposition (SVD) of the equilibrium matrix [22, 23]. Only six kinematic constraints are considered in the equilibrium-matrix study in order to avoid rigid body displacements. The square module studied in this paper has one infinitesimal mechanism and seven independent self-stress states. For six kinematic constraints on displacements, the bridge has 31 elementary self-stress states and one infinitesimal mechanism.

\section{Design specifications}

A pedestrian bridge composed of tensegrity ring modules is required to be over a highway in the region of Lausanne, Switzerland. A span of $20 \mathrm{~m}$ and a distance from the ground of $3 \mathrm{~m}$ are assumed. The bridge geometry is chosen such that it has the minimum internal space required for two pedestrians to walk side-by-side (Figure 4). This space can be represented by a rectangle with a height of $2.5 \mathrm{~m}$ and a width of $1.3 \mathrm{~m}$. The bridge is composed of four identical tensegrity modules. Each tensegrity ring module for the bridge is assumed to be $5 \mathrm{~m}$ long. Symmetry about midspan is obtained by mirroring two modules about plane Z-Z (Figure 4).

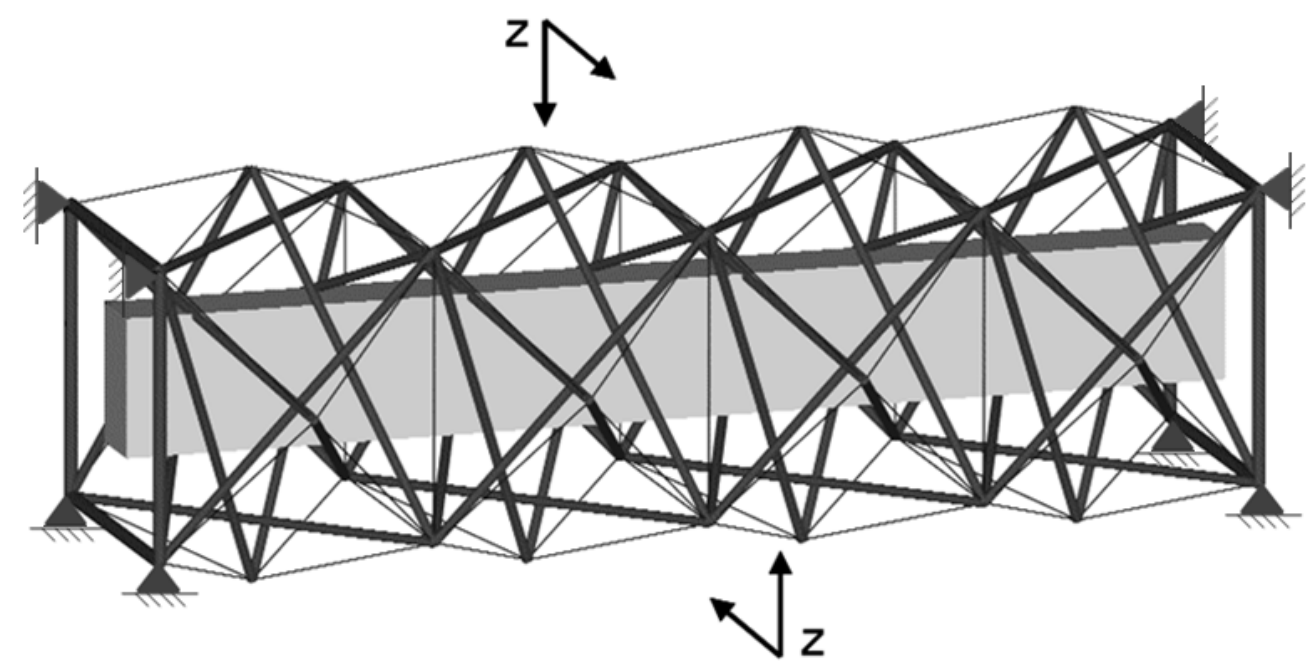

Figure 4: Tensegrity square bridge 
Using the method presented in Section 2, various geometries can be generated. The following geometry is found to satisfy the requirement for an internal space of $1.3 \mathrm{~m} \times 2.5 \mathrm{~m}$ :
a. $\quad$ length of layer cable $=520.0 \mathrm{~cm}$
b. $\quad$ length of $\mathrm{x}$-cable $=376.4 \mathrm{~cm}$
c. length of diagonal strut $=721.4 \mathrm{~cm}$
d. length of non-diagonal strut $=724.0 \mathrm{~cm}$

The above member dimensions are constant for the whole study. The cables and the struts of the bridge are assumed to be made of steel. Nodes at both extremities of the structure are fixed in all three directions.

The bridge is required to meet the norms for safety and serviceability specified by the Swiss codes SIA 260 and 263 [24, 25]. Safety criteria ensure that there is sufficient strength to avoid failure and instabilities. Buckling strength governs the design of struts. The buckling strength $N_{k}$ of struts is given by:

$$
N_{k}=\chi_{k} \frac{f_{y} A}{1.05}
$$

$\chi_{k}$ is the reduction factor for buckling, $f_{y}$ is the yield strength and $A$ is the cross-section area of the cable. A slenderness limit is also specified for the struts. The slenderness ratio $\lambda_{k}$ should be less than 200. Cable cross-sections are governed by tensile strength requirements. The available tensile strength $T$ is calculated using the following formula:

$$
T=\frac{f_{y} A}{1.05}
$$

$f_{y}$ is the yield strength in $\mathrm{kN} / \mathrm{cm}^{2}$ and $A$ is the cross-section of the cable in $\mathrm{cm}^{2}$.

Serviceability criteria ensure that the structure is able to accomplish its function. For a bridge limits on vertical displacements are prescribed for functionality, comfort and appearance. All three criteria are evaluated in terms of the length of the bridge. The functionality and appearance criteria are the most limiting with acceptable displacements less than length/700. The three criteria are evaluated at the two bottom nodes at midspan as the largest displacements are observed at these nodes. Another constraint that affects the bridge design is the ratio between the diameter and the thickness for tubular struts. This ratio must be less than 50 to avoid local instabilities.

Two independent live load models are employed for a pedestrian bridge: one with a uniform load and another with a concentrated center load. The values of vertical loads (dead load and service load) and their corresponding load factors are presented in Table 1. Load factors are used to accommodate uncertainties in load values and modeling assumptions. 
Table 1: Loads on the structure

\begin{tabular}{llll}
\hline Load on the structure & $\begin{array}{l}\text { Load factor } \\
\text { Concentrated load }\end{array}$ & Uniform load & \\
\hline Dead load & 1.35 & 1.35 & $\gamma=78.5 \mathrm{kN} / \mathrm{m}^{3}$ \\
Service load: concentrated & 1.50 & 0.00 & $15 \mathrm{kN}$ \\
Service load: uniform & 0.00 & 1.50 & $4 \mathrm{kN} / \mathrm{m}^{2}$ \\
\hline
\end{tabular}

These loads are applied on a walking path that is composed of a steel deck. The deck transmits the loads to the four base nodes of each module. The walking path is $1.3 \mathrm{~m}$ wide and $20 \mathrm{~m}$ long. Considering the above loads and partial factors the uniform load model produces the largest displacements at midspan and the largest forces in the members. Thus, it is taken to be the critical load model for the design of the structure. For the dimensions of this structure and the critical load model with the respective partial factors, nodal forces of $12 \mathrm{kN}$ are estimated at the bottom nodes of both extremities and $24 \mathrm{kN}$ are estimated at the intermediate bottom nodes.

The structure is analyzed using the dynamic relaxation method with kinematic damping. Dynamic relaxation is an iterative method that traces the motion of the structure from the moment of loading using an analogy of damped vibration [26]. Due to the presence of fictitious damping, the structure reaches static equilibrium. The dynamic relaxation method is advantageous for geometrically nonlinear and flexible structures since it does not require the inversion of the stiffness matrix and also determines loaded geometry and element stresses in one step. Dynamic relaxation may also be used to determine the number of infinitesimal mechanisms and the number of independent states of self-stress for the tensegrity bridge. The bridge has 48 self-stress states and no infinitesimal mechanisms when all boundary nodes are blocked (Figure 4). Consequently, the structure is statically indeterminate and kinematically determinate.

\section{Cost model}

In this study, a cost model is used that reflects the total cost $C$ of assembling the whole structure. This model includes two parts: the cost of elements $c_{s}$ and the cost of joints $c_{j}$.

$$
C=c_{s}+c_{j}
$$

The cost of elements can be further separated into the cost of struts $\left(c_{s, s}\right)$ and the cost of cables $\left(c_{s, c}\right)$. Struts are made out of steel hollow tubes. Data obtained from local steel construction companies indicate that the price of hollow tubes varies according to its diameter and its thickness. The following linear equation has been obtained using linear regression on this data for calculating the cost of hollow tubes:

$$
c_{s, s}=74.84 \cdot A_{s}
$$


$c_{s, c}$ is the cost per unit length in $\mathrm{CHF} / \mathrm{m}$ and $A_{s}$ is the area of the cross-section in $\mathrm{cm}^{2}$. For cables, the cost varies with cross-section area and length. The following equation relating cost per unit length to the area has been obtained using regression:

$$
c_{s, c}=67.34 \cdot A_{c}^{0.712}
$$

$c_{s, c}$ is the cost in $\mathrm{CHF} / \mathrm{m}$ and $A_{c}$ is the area of the cross-section in $\mathrm{cm}^{2}$.

The second component in Equation 3 and potentially, the most important factor affecting the total cost of the structure, is the cost of the joints. In steel construction joints are very expensive details that may determine other aspects of the design of a structure. In this study, only a single topology is analyzed. Therefore, the number of joints and hence, the cost of joints is assumed to remain constant for all design solutions.

\section{Design using parametric analysis and traditional design}

Engineers generally adopt an iterative approach that is similar to a gradient-based search to design structures. The initial design often violates design constraints. Depending upon the nature of constraint violations, larger member sizes are used and then verified for other criteria. Such an approach is simulated using parametric studies. Parametric studies are conducted to understand the individual influence of each variable on the responses related to the design constraints. The goal is to identify the optimal direction, similar to the steepest slope in a gradient-based search. The effects of parameters on the vertical displacement at midspan and the maximum compressive force are evaluated. The vertical displacement at midspan reflects serviceability criteria, while the maximum compressive force is related to failure due to buckling. The vertical displacement is maximum at the two lower nodes at midspan named A and B (see Figure 5).

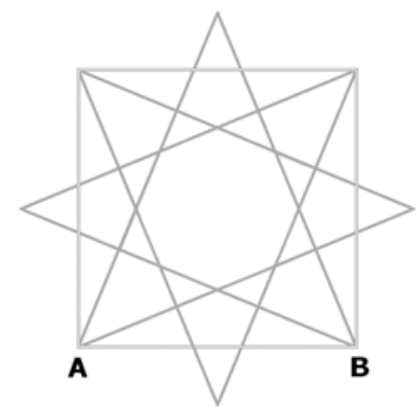

Figure 5: View from section Z-Z (Figure 4)

Maximum compressive forces are determined in struts $\mathrm{X}$ and $\mathrm{Y}$. Strut $\mathrm{X}$ refers to a diagonal strut in each of the two outer modules, while $\mathrm{Y}$ is a non-diagonal strut in each of the two central modules. These two elements have the highest internal compressive forces.

The influence of the following five parameters on vertical deflection and maximum compressive force is examined:

- cross-section area of x-cables

- cross-section area of layer cables

- cross-section area of struts 
- $\quad$ self-stress

- ratio between the stiffness of struts and cables (varying the Young's modulus of struts).

For all the parametric studies, a base design configuration is assumed. The values for this configuration are inspired from previous research [27-29]. The parameter of interest is alone varied while the values for other parameters are left unchanged from the base configuration. Specifically, the parameter of interest is incremented in steps and two responses, displacement at midspan and maximum compressive force, are evaluated. Details of the base configuration are given in Table 2.

Table 2: Details of the base square module

\begin{tabular}{llll}
\hline & Struts & X-cables & Layer cables \\
\hline $\mathrm{A}\left[\mathrm{cm}^{2}\right]$ & 11.10 & 0.28 & 0.28 \\
$\mathrm{E}\left[\mathrm{kN} / \mathrm{cm}^{2}\right]$ & 21000 & 11500 & 11500 \\
\hline
\end{tabular}
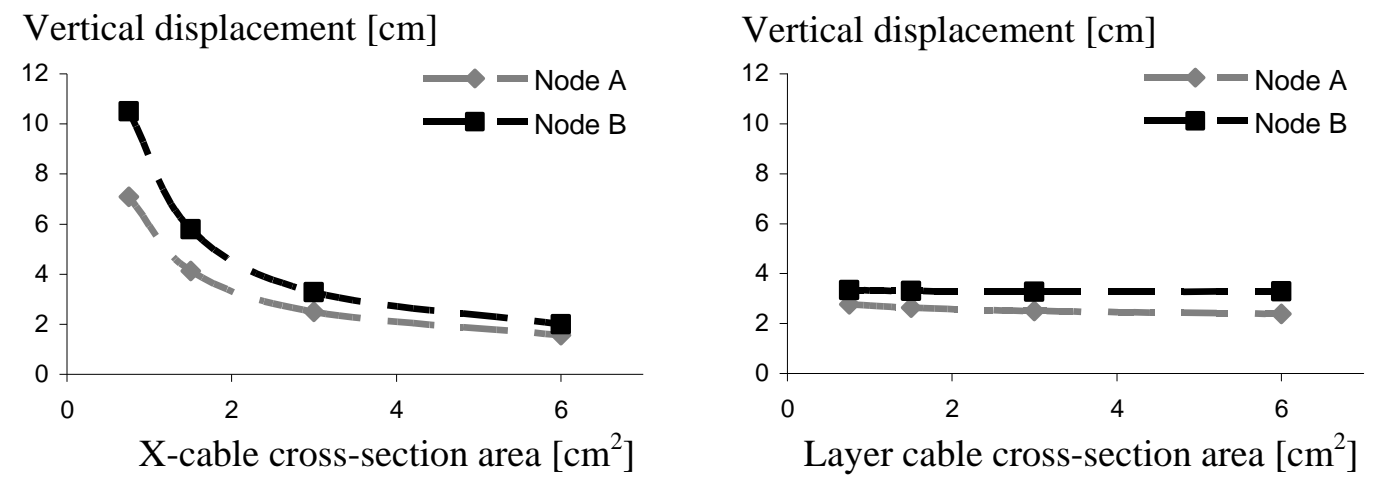

Figure 6: Influence of the cross-sectional area of x-cables and layer cables on the vertical displacement at midspan

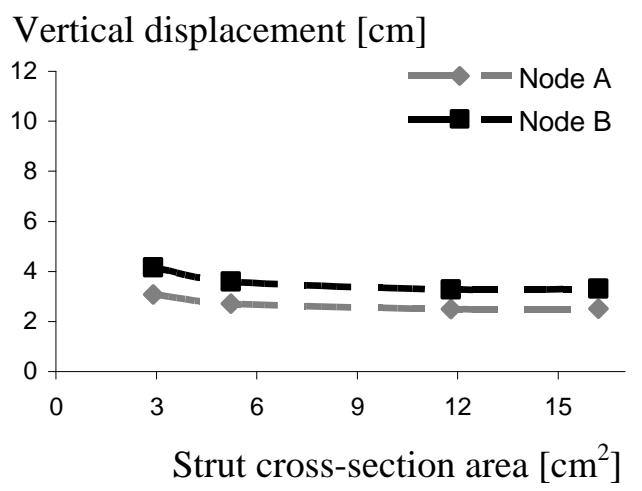

Figure 7: Influence of the cross-sectional area of struts on the vertical displacement at midspan 


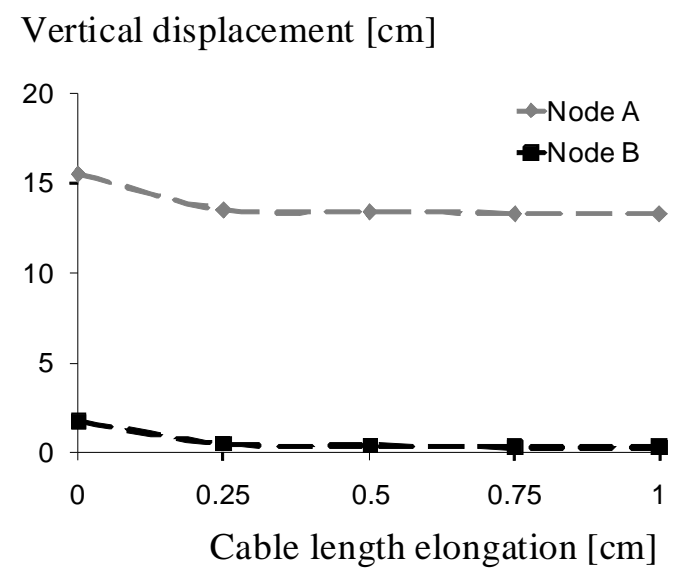

Figure 8: Influence of self-stress on the vertical displacement at midspan

The parametric analysis reveals that the cross-sectional area of the x-cables is the parameter with the most influence on the vertical displacement (Figure 6). Doubling the cross-section area of these cables significantly increases the total rigidity of the system and thus decreases vertical displacements. Figure 6 shows also that areas of layer cables have little influence on vertical displacements. However, when the cross-sectional area of struts is increased displacements initially decrease and then remain almost constant (Figure 7). Figure 8 shows that increasing the level of self-stress initially increases the rigidity of the structure by a small amount. However, for large values of self-stress, there is almost no change in vertical displacement.

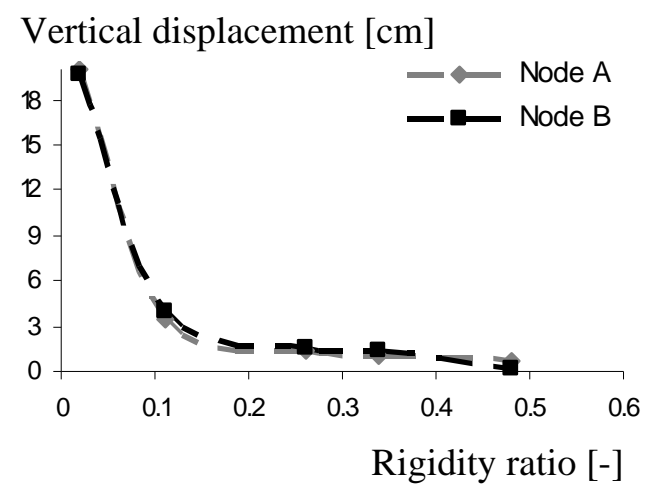

Figure 9: Influence of the stiffness ratio between bars and cables on the vertical displacement at midspan

The influence of the ratio between the stiffness of bars and cables on vertical displacements is shown in Figure 9. The plot shows that the decrease in vertical displacement is negligible for values of the ratio greater than 0.2 . Values for the stiffness ratio between bars and cables should thus be approximately situated within 0.1 and 0.2 for a good design tradeoff with respect to displacements.

The magnitudes of the vertical displacements at the two nodes at midspan are different in all Figures. This means that the structure twists. This behavior is explained by the asymmetric geometry of the structure. Asymmetry is caused by the helical circuits created by the arrangement of the diagonal struts around the longitudinal axis of the bridge. The torsional 
behavior decreases when the cross-section area of the x-cables is increased (Figure 6). On the other hand, it increases with the cross-sectional area of the layer cables (Figure 6).

Normalized compressive force [-]

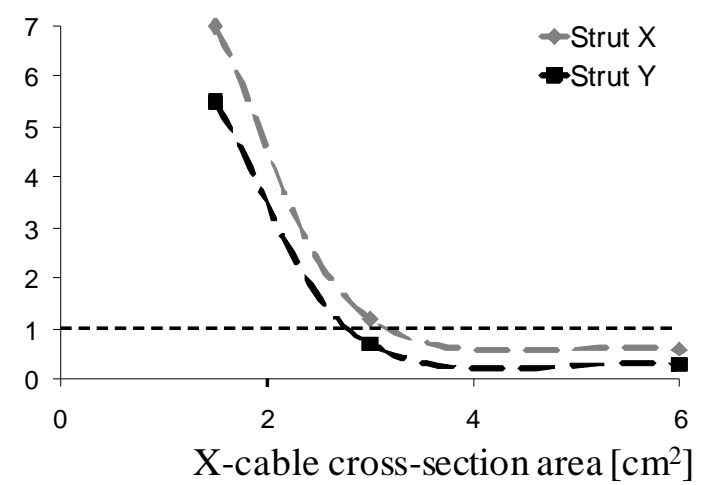

Normalized compressive force [-]

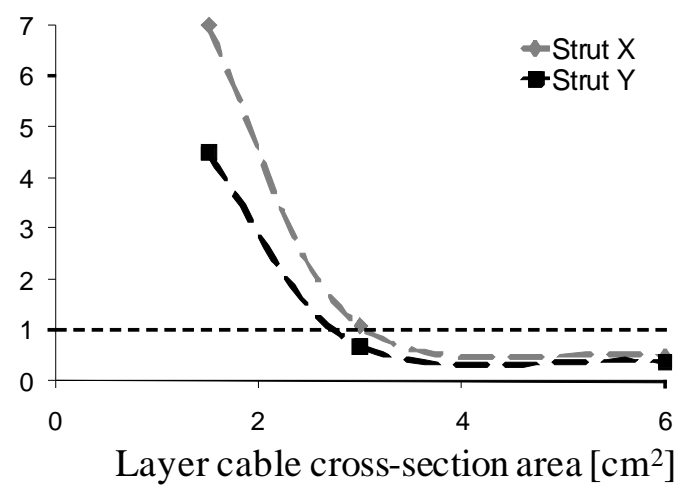

Figure 10: Influence of the cross-sectional area of $\mathrm{x}$-cables and layer cables on the compressive force normalized to the buckling capacity

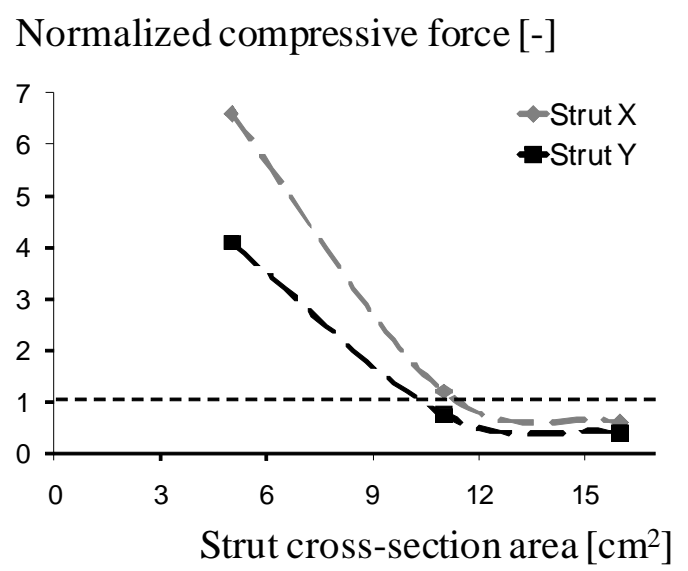

Figure 11: Influence of the cross-sectional area of struts on maximum compressive forces

Increasing the cross-sectional area of both x-cables and layer cables decreases the ratio of maximum compressive force to buckling strength (Figure 10). However, adjusting the crosssection area of $\mathrm{x}$-cables is preferable since forces are more uniformly distributed amongst the struts. Figure 11 reflects Euler's law that the ratio of maximum compressive force to buckling strength decreases exponentially with increases in the cross-sectional area of struts.

A feasible configuration is found using trends from the parametric analyses. Values for the parameters are modified in steps to meet the design constraints. The first parameter that is modified is the area of the $\mathrm{x}$-cables. A value for the area of the $\mathrm{x}$-cables is found for which both vertical displacements and tensile strength constraints are satisfied. The next variable considered is the area of the struts. A tube with sufficient cross-section area and second moment of area is chosen such that it avoids the instability due to buckling. Finally, self-stress is introduced in 
order to eliminate slack cables and increase slightly the stiffness of the structure. At every step of the procedure the dynamic relaxation method is used to analyze the bridge. The details of the final solution are given in Table 3.

Table 3: Design found using parametric analyses

\begin{tabular}{lccc}
\hline Characteristic & Struts & Layer cables & X-cables \\
\hline $\mathrm{L}[\mathrm{cm}]$ & 723.95 & 519.60 & 376.10 \\
$\mathrm{D}[\mathrm{cm}]$ & 10.16 & 1.90 & 2.25 \\
$\mathrm{~A}\left[\mathrm{~cm}^{2}\right]$ & 15.20 & 3.00 & 4.00 \\
$\mathrm{E}\left[\mathrm{kN} / \mathrm{cm}^{2}\right]$ & 21000 & 11500 & 11500 \\
\hline
\end{tabular}

The dead load of the structure is $51 \mathrm{kN}$. The level of self-stress in terms of the elongation in length is $0.40 \mathrm{~cm}$ for layer cables and $0.36 \mathrm{~cm}$ for $\mathrm{x}$-cables. Based on the cost model the material cost for this bridge configuration (joints not included) is estimated to be 89 '700 CHF.

\section{Design optimization through stochastic search}

Structural design is an inverse (abductive) task in which engineers search for solutions given required functionality and behavior. Parametric studies are an engineering approach for obtaining design solutions as there is seldom any closed form mathematical expression for determining designs directly from specified structural behavior. The design that is proposed by parametric studies satisfies all design constraints. However, this solution may not be an optimal solution. It may simply be a local minimum in a very large and complex solution space. Plots from parametric studies show the influence of a single parameter only. Implicitly the assumption in parametric studies is that the general trends are valid even when the initial design configuration is altered. This assumption is often false.

Global optimization techniques, such as stochastic search, are powerful techniques for complex engineering tasks. They find solutions that have a greater chance of being the global minimum than solutions provided by parametric analysis. Another advantage is objectives can be altered easily within the search procedure to study effects of different requirements and priorities.

The method used for stochastic search and optimization here is PGSL (Probabilistic Global Search Lausanne). The principal assumption of this method is that sets of near-optimal solutions will be found near sets of good solutions [18]. The PGSL algorithm is based on a probability density function that is iteratively modified so that more exhaustive searches are made in regions of good solutions. This method has been successfully used to reduce cost in timber housing design [20].

The first step is to identify the parameters to be included in the algorithm. The following six parameters are included in this study:

1. area of layer cables $\left[0.05 ; 10 \mathrm{~cm}^{2}\right]$

2. area of $\mathrm{x}$-cables $\left[0.05 ; 10 \mathrm{~cm}^{2}\right]$ 
3. outer diameter of tubular struts [2; $14 \mathrm{~cm}]$

4. diameter to thickness ratio of tubular struts [5; 50]

5. self-stress in layer cables $[0 ; 1 \mathrm{~cm}]$

6. self-stress in $\mathrm{x}$-cables $[0 ; 1 \mathrm{~cm}]$

The above variables are mutually independent and they have an important impact on the performance of the system. The numbers within rectangular brackets beside each parameter indicate lower and upper bounds of possible values. For self-stress, the numbers in brackets correspond to elongations in the respective cables. In this study, element topology and span of the bridge are assumed to be fixed.

The struts have a tubular cross-section and hence there are two parameters associated with their section characteristics: the outer diameter and the thickness. Since there is an upper bound on the slenderness limit, there is a dependency between these two parameters. Therefore, this limit is explicitly modeled by including the slenderness ratio as a parameter and constraining it to be less than 200.

For this study, the objective function consists of two components: (1) the cost $(C)$ of the structure including joints as given in Equation 3, and (2) penalty costs $(P)$ that account for each constraint violation.

$$
O F=C+P
$$

There is often a tradeoff between the two components of the objective function. For example, decrease in the cost $C$ may result in constraint violations and increase the value of the objective function through the penalty function $P$.

The penalties for the violation of constraints are calculated as the additional costs that are likely to be incurred to force the solution to satisfy the constraints. There are four penalty components as described in Equation 7.

$$
P=P_{d}+P_{t}+P_{c}+P_{s c}
$$

The penalty cost $P_{d}$ corresponds to the cost that is estimated to reduce displacements so that the solution satisfies displacement criteria. $P_{t}$ and $P_{c}$ correspond to the penalty costs that are estimated to make the design solution satisfy the tensile and compressive stress limits. An additional penalty $P_{s c}$ is considered to avoid slack cables in the structure.

Parametric studies have shown that the x-cables are the most important load bearing component in the structure. Thus, $P_{d}$ is calculated as the cost corresponding to the additional x-cable area required to reduce vertical displacements to the allowable limit. From the parametric study (Figure 6) on the variation of vertical displacement $(\delta)$ in relation to the area of x-cables $(A)$, the following equation is obtained:

$$
A=5.16 \cdot \delta^{-1.23}
$$

While Figure 6 is exact only for a parametric study using the solution given in Table 2, the same general trend is also observed for other solutions. Therefore, this penalty, which is only an approximation of the cost required to compensate excess displacement is valid.

For a given solution with vertical displacement $\delta, P_{d}$ is calculated using Equation 9. 


$$
P_{d}=\left\{\begin{array}{l}
0, \text { if } \delta<\delta_{0}=2.85 \mathrm{~cm} \\
C\left(A_{0}\right)-C(A), \text { if } \delta>\delta_{0}
\end{array}\right.
$$

$A_{0}$ is the estimated minimum cross-sectional area that is required to keep vertical displacements under the allowable limit $\delta_{0}$. $A$ is the cross-section area corresponding to the evaluated vertical displacement $\delta . A$ and $A_{0}$ are calculated using Equation 8. Equation 5 is used to determine the cost for a given area.

The penalty costs for excessive stress are also estimated. If tensile stresses exceed the tensile capacity of the cables, a penalty cost is estimated for the additional cable area required to take the calculated force according to Equation 5. For the struts, if compressive stresses exceed their buckling strength, a penalty cost is estimated for the additional area necessary to prevent buckling using Equation 4.

PGSL requires upper and lower limits for each input parameter. It samples values for each parameter within these bounds. PGSL provides consistently good results for a number of evaluations greater than 4000 . The details of the minimum cost solution obtained using PGSL are given in Table 4. This value may not correspond exactly to the global optimum since this is identified precisely only through the evaluation of all possible solutions.

Table 4: Final solution generated by PGSL

\begin{tabular}{llll}
\hline Characteristic & Struts & Layer cables & X-cables \\
\hline $\mathrm{L}[\mathrm{cm}]$ & 723.95 & 519.45 & 376.13 \\
$\mathrm{D}[\mathrm{cm}]$ & 11.21 & 1.60 & 1.90 \\
$\mathrm{~A}\left[\mathrm{~cm}^{2}\right]$ & 1020 & 2.21 & 3.00 \\
$\mathrm{E}\left[\mathrm{kN} / \mathrm{cm}^{2}\right]$ & 21000 & 11500 & 11500 \\
\hline
\end{tabular}

The dead load of the structure is $31.25 \mathrm{kN}$. The level of self-stress in terms of the elongation in length is $0.55 \mathrm{~cm}$ for layer cables and $0.30 \mathrm{~cm}$ for $\mathrm{x}$-cables. Based on the cost model the material cost for this bridge configuration (joints not included) is estimated to be 53'300 CHF. Table 5 presents results from 10 PGSL runs. It shows that PGSL consistently finds solutions that are less expensive than the solution found using parametric study. 
Table 5: Solutions from 10 PGSL runs

\begin{tabular}{llllll}
\hline Run & $\begin{array}{l}\text { Area of layer } \\
\text { cable }\left[\mathrm{cm}^{2}\right]\end{array}$ & $\begin{array}{l}\text { Area of x- } \\
\text { cable }\left[\mathrm{cm}^{2}\right]\end{array}$ & $\begin{array}{l}\text { Diameter of } \\
\text { strut }[\mathrm{cm}]\end{array}$ & $\begin{array}{l}\text { Diameter to } \\
\text { thickness ratio }\end{array}$ & Cost [CHF] \\
\hline 1 & 2.41 & 4.00 & 11.20 & 47.38 & $55^{\prime} 400$ \\
2 & 2.32 & 5.89 & 11.02 & 46.71 & $60^{\prime} 500$ \\
3 & 2.21 & 3.00 & 11.21 & 46.94 & $53^{\prime} 300$ \\
4 & 3.55 & 4.09 & 11.28 & 47.39 & $57^{\prime} 300$ \\
5 & 2.28 & 3.95 & 11.25 & 47.95 & $55^{\prime} 000$ \\
6 & 3.05 & 4.10 & 11.17 & 47.59 & $55^{\prime} 900$ \\
7 & 2.10 & 3.90 & 11.34 & 48.64 & $54^{\prime} 700$ \\
8 & 2.00 & .3 .32 & 11.21 & 47.12 & $53^{\prime} 600$ \\
9 & 2.78 & 3.14 & 11.27 & 48.14 & $53^{\prime} 700$ \\
10 & 2.64 & 3.84 & 11.18 & 48.74 & $54^{\prime} 000$ \\
\hline
\end{tabular}

Table 6 gives the minimum, the maximum and the average values for solution parameters from the 10 PGSL runs. It also shows the standard deviation for each parameter.

Table 6: Summary of results from PGSL

\begin{tabular}{lllll}
\hline Characteristic & $\begin{array}{l}\text { Minimum } \\
\text { value }\end{array}$ & $\begin{array}{l}\text { Maximum } \\
\text { value }\end{array}$ & $\begin{array}{l}\text { Average value } \\
(\mathrm{n}=10)\end{array}$ & Standard deviation \\
\hline $\begin{array}{l}\text { Area of layer } \\
\text { cable }\left(\mathrm{cm}^{2}\right)\end{array}$ & 2.00 & 3.05 & 2.72 & 0.72 \\
$\begin{array}{l}\text { Area of x-cable } \\
\left(\mathrm{cm}^{2}\right)\end{array}$ & 3.01 & 5.89 & 3.93 & 0.58 \\
$\begin{array}{l}\text { Diameter of } \\
\text { struts (cm) }\end{array}$ & 11.02 & 11.34 & 11.21 & 0.10 \\
$\begin{array}{l}\text { Diameter to } \\
\text { thickness ratio }\end{array}$ & 46.71 & 48.64 & 47.66 & 0.67 \\
$\begin{array}{l}\text { Cost [CHF] } \\
5\end{array}$ & 53 ’300 & 60 ’500 & 55 '300 & 2 '100 \\
\hline
\end{tabular}

Table 6 shows that the diameter of struts is the parameter with the smallest standard deviation. Other parameters such as the cross-section area of $\mathrm{x}$-cables and layer cables have a larger variability than the diameter. Finally, although values for design parameters vary significantly 
between PGSL runs, the costs of the final solutions remain consistently close to CHF 55'000. Figure 12 shows the convergence to the best solution in a sample run of PGSL.

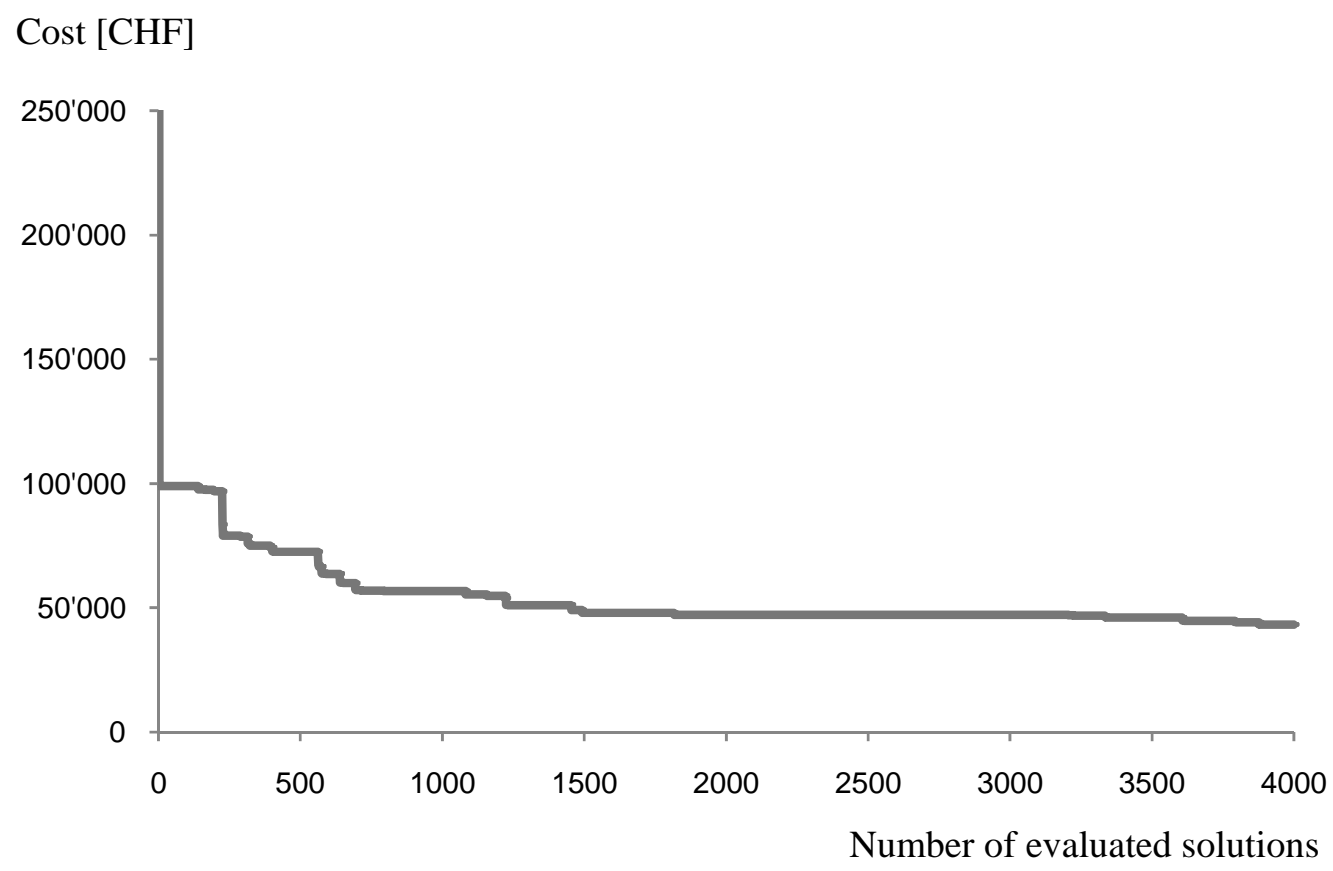

Figure 12: Plot showing PGSL convergence in terms of the cost of the best solution found after evaluating each sample

\section{Discussion}

The two design methods studied in this paper result in feasible solutions that meet the design criteria. However, the solution from parametric studies is more expensive compared with the solution obtained using PGSL. Solutions are compared in Table 7.

Table 7: Comparison of the solutions resulting from use of the two methods

\begin{tabular}{lll}
\hline Study & Stochastic search & Parametric analyses \\
\hline Cost (CHF) & $533^{\prime} 300$ & $89^{\prime} 700$ \\
Dead Load (kN) & 31.25 & 51 \\
Avg. Disp. (cm) & 0.86 & 0.78 \\
Slenderness ratio & 186 & 211 \\
\hline
\end{tabular}

The PGSL solution has the advantage of being lighter. The vertical displacement at midspan is, however, larger for the PGSL solution. The best solution using PGSL has a cost that is $40 \%$ lower than the cost of the solution resulting from parametric analyses. 
Another observation is that solutions generated using PGSL usually have cross-section properties that are not readily available in practice. There are two solutions for such situations: requesting a special order or choosing sections closest to the ones proposed by PGSL. A special order will however increase the cost of the structure. On the other hand, changing member sizes to nearest standard sections can result in the violation of a constraint as solutions from PGSL may be sensitive to the parameters. Such shortcomings can be overcome if design parameters are modeled as discrete variables.

The performance of PGSL over parametric analyses can be explained considering parametric plots for two starting solutions. Plots in Figure 13 show the influence of the cross-sectional area of struts on the average vertical displacement at midspan for two configurations. Configuration I uses the following parameter values: $A_{\text {layer cables }}=4.00 \mathrm{~cm}^{2}$ and $A_{x \text {-cables }}=6.00 \mathrm{~cm}^{2}$; for configuration II: $\mathrm{A}_{\text {layer cables }}=1.00 \mathrm{~cm}^{2}$ and $\mathrm{A}_{x \text {-cables }}=2.00 \mathrm{~cm}^{2}$. Both configurations show similar trends: the vertical displacement first decreases, reaches a minimum and then increases. However, the minimum occurs at different points for the two cases and the gradients of the curves also vary. For the first configuration the minimum corresponds to a strut section of approximately $12 \mathrm{~cm}^{2}$, while for the second it is found at approximately $5 \mathrm{~cm}^{2}$. Plots from parametric studies reflect only a small part of a large solution domain. Parametric analyses remain valid. However, they may not lead to near optimal solutions for tensegrity systems especially if the configurations are not similar. On the contrary, PGSL samples all design parameters and is not dependent on the starting solution. Therefore, it avoids local minima and the chances of finding the true global optimum are increased.

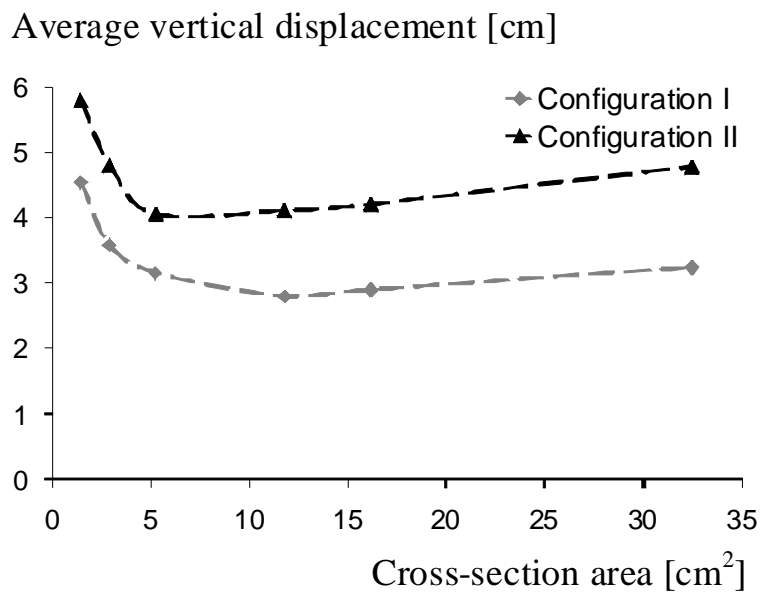

Figure 13: Trends for two configurations with the same cross-sectional area of struts, configuration I: $\mathrm{A}_{\text {layer cables }}=4.00 \mathrm{~cm}^{2}, \mathrm{~A}_{\mathrm{x} \text {-cables }}=6.00 \mathrm{~cm}^{2}$; configuration II: $\mathrm{A}_{\text {layer cables }}=1.00 \mathrm{~cm}^{2}, \mathrm{~A}_{\mathrm{x} \text {-cables }}=2.00 \mathrm{~cm}^{2}$

PGSL finds a better solution than parametric study for the following reasons:

1. Stochastic search is a global search method while parametric studies evaluate only a part of the solution space. PGSL samples the entire solution space and gradually focuses around regions with good solutions. However, parametric studies utilize gradients and their performance is determined by the initial trial solution.

2. An important difference between the two methods is that self-stress is modeled as a variable in the stochastic search, while a constant self-stress is considered for parametric design. Finding a feasible solution using parametric studies is difficult when including self-stress as a parameter. 
3. Solutions from stochastic search attempt to satisfy all design constraints. On the other hand, solutions from parametric study tightly satisfy only the buckling constraint while staying well under the limits for displacement and tensile strength.

Examination of the system behavior reveals characteristics of the two continuous circuits of diagonal struts that run across the span. These circuits may carry tensile stresses greater than 30 $\mathrm{N} / \mathrm{mm}^{2}$. Consequently, such stresses may increase the cost of joints. Stochastic search can also be used for finding solutions such that the struts carry little or no tension, thereby allowing for less expensive joints. In this case an additional constraint is added to the problem. The solutions found for tensionless struts require the area of both cables to be increased by large amounts (4.5 $-7.0 \mathrm{~cm}^{2}$ ) and hence include a large increase in the material cost. It also leads to high values for the maximum compressive stresses in the struts. Therefore such solutions can only be justified when the saving in joint costs are substantial.

\section{Conclusions}

This paper focuses on the use of stochastic search for the design with minimum cost of a pedestrian bridge made of square hollow-rope tensegrity ring modules. Two design methods are compared with the aim of finding the minimal cost solution. The first method attempts to simulate the practice in design offices using parametric analyses. The second method uses PGSL, a stochastic search algorithm. The challenge of designing a tensegrity structure for minimum cost is combinatorial. Therefore millions of possibilities exist. Erection costs are assumed to be the same for all solutions, and life cycle costing is not included in this study.

The conclusions from this study are as follows:

- Results from parametric analyses show that certain cables (x-cables) are the fundamental load-bearing elements in these tensegrity bridges. Their stiffness has the largest effect on maximum compressive forces and vertical displacements.

- Both parametric analysis and stochastic search generate designs that satisfy safety and serviceability criteria.

- The best solution using stochastic search has a cost that is $40 \%$ lower than that of the solution from parametric analysis.

- Parametric analyses are useful to obtain a broad understanding of the influence of each parameter. Results from parametric studies can also help in defining effective penalty costs for enforcing constraints during global optimization.

- Stochastic search can support what-if analyses as engineers can easily modify objective function to accommodate additional constraints. For example, results show that dead weight increases significantly when tensionless struts are required.

These results underline the complexity of the design of tensegrity structures and the efficiency of advanced computing methods. Work in progress includes studies of more elaborate cost models and a representation that models design parameters as discrete variables. Additionally, a prototype of the tensegrity bridge will be built and studied experimentally.

\section{Acknowledgments}

Authors would like to thank the Swiss National Science Foundation for supporting this work. They are also grateful to Prof. René Motro and his research team at LMGC (Université de Montpellier II). N. Bel Hadj Ali is thanked for discussion and advice. 


\section{References}

[1] Perlberg D. Snelson and structure. Artforum 1977.

[2] Pugh A. An Introduction to Tensegrity: University of California Press 1976.

[3] Motro R, ed. Tenségrité: Hermes Science 2005.

[4] Tibert AG, Pellegrino, S. Deployable Tensegrity Masts. 44th AIAA/ASME/ASCE/AHS/ASC Structures, Structural Dynamics, and Material Conference and Exhibit. Norfolk, VA 2003.

[5] Tibert AG, Pellegrino S. Review of form-fiding methods for tensegrity structures. International Journal of Space Structures. 2003;18(4).

[6] Masic M, Skelton RE, Gill PE. Optimization of tensegrity structures. International Journal of Solids and Structures. 2006;43(16):4687-703.

[7] Fuller RB. Synergetics: Explorations in the Geometry of Thinking. New York: MacMillan Publishing Co., Inc, 1975.

[8] Kebiche K, Kazi-Aoual MN, Motro R. Geometrical non-linear analysis of tensegrity systems. Engineering Structures. 1999;21(9):864-76.

[9] Quirant J, Kazi-Aoual MN, Motro R. Designing tensegrity systems: the case of a double layer grid. Engineering Structures. 2003;25(9):1121-30.

[10] Arora JS, Elwakeil OA, Chahande AI, Hsieh CC. Global optimization methods for engineering applications: A review. Structural and Multidisciplinary Optimization. 1995;9(3):137-59.

[11] Kicinger R, Obayashi S, Arciszewski T. Evolutionary multiobjective optimization of steel structural systems in tall buildings. In: Obayashi S, Deb K, Poloni C, Hiroyasu T, Murata T, eds. Evolutionary Multi-Criterion Optimization, Proceedings. Berlin: Springer-Verlag Berlin 2007:604-18.

[12] Degertekin SO, Saka MP, Hayalioglu MS. Optimal load and resistance factor design of geometrically nonlinear steel space frames via tabu search and genetic algorithm. Eng Struct. 2008 Jan;30(1):197-205.

[13] Camp CV, Bichon BJ. Design of space trusses using ant colony optimization. J Struct Eng-ASCE. 2004 May;130(5):741-51.

[14] Shea K, Smith IFC. Improving Full-Scale Transmission Tower Design through Topology and Shape Optimization. Journal of Structural Engineering. 2006;132(5):781-90.

[15] Domer B, Fest E, Lalit V, Smith IFC. Combining Dynamic Relaxation Method with Artificial Neural Networks to Enhance Simulation of Tensegrity Structures. Journal of Structural Engineering. 2003;129(5):672-81.

[16] Domer B, Raphael B, Shea K, Smith IFC. A Study of Two Stochastic Search Methods for Structural Control. Journal of Computing in Civil Engineering. 2003;17(3):132-41.

[17] Paul C, Lipson H, Cuevas FV. Evolutionary form-finding of tensegrity structures. Genetic and Evolutionary Computation Conference. Washington D.C. 2005:3-10.

[18] Raphael B, Smith IFC. A direct stochastic algorithm for global search. Applied Mathematics and Computation. 2003;146(2-3):729-58.

[19] Shea K, Fest E, Smith IFC. Developing intelligent tensegrity structures with stochastic search. Advanced Engineering Informatics. 2002;16(1):21-40.

[20] Svenerudh P, Raphael B, Smith IFC. Lowering Costs of Timber Shear-Wall Design using Global Search. Engineering with Computers. 2002;18(2):93-108.

[21] Motro R, Maurin B, Silvestri C. Tensegrity Rings and the Hollow Rope. IASS Symposium 2006, New Olympics, New Shells and Spatial Structures. Beijing 2006:470-1.

[22] Pellegrino S, Calladine CR. Matrix analysis of statically and kinematically indeterminate frameworks. International Journal of Solids and Structures. 1986;22(4):409-28. 
[23] Nishimura Y, Murakami H. Initial shape-finding and modal analyses of cyclic frustum tensegrity modules. Computer Methods in Applied Mechanics and Engineering. 2001;190(4344):5795-818.

[24] SIA. Steel Construction. Zurich: Swiss Society of Engineers and Architects 2003.

[25] SIA. Basis of Structural Design. Zurich: Swiss Society of Engineers and Architects 2003.

[26] Barnes MR. Form Finding and Analysis of Tension Structures by Dynamic Relaxation. International Journal of Space Structures. 1999;14:89-104.

[27] Fest E, Shea K, Smith IFC. Active Tensegrity Structure. Journal of Structural Engineering. 2004;130(10):1454-65.

[28] Adam B, Smith IFC. Reinforcement Learning for Structural Control. Journal of Computing in Civil Engineering. 2008;22(2):133-9.

[29] Adam B. Adaptive Civil Engineering Structures. Thesis No 3750, EPFL 2007. 